\title{
Spectral analysis of type II supernovae
}

\author{
Claudia P. Gutiérrez ${ }^{1}$, Joseph Anderson ${ }^{1}$, Mario Hamuy ${ }^{1}$, \\ Gastón Folatelli ${ }^{2}$ and the CSP team ${ }^{3}$ \\ ${ }^{1}$ Departamento de Astronomía Universidad de Chile, \\ Camino el Observatorio 1515, Santiago, Chile \\ email: cgutierr@das.uchile.cl \\ ${ }^{2}$ Institute for the Physics and Mathematics of the Universe (IPMU) University of Tokyo, \\ 515 Kashiwanoha Kashiwa, Chiba 2778583, Japan \\ ${ }^{3}$ Carnegie/Las Campanas Observatories
}

\begin{abstract}
We present spectroscopic analysis of 63 type II supernovae. We present preliminary results on correlations between spectroscopic and photometric properties, focusing on light-curve decline rates, absolute magnitudes and $H_{\alpha}$ lines profiles. We found the ratio of absorption to emission of $H_{\alpha}$ P-Cygni profile as the dominant measured parameter as it has the highest median correlation with all other parameters.
\end{abstract}

Keywords. Supernova, Spectra, Photometry

\section{Introduction}

Type II Supernovae (SNe II) were initially classified in two subclasses depending to the shape of the light curve. SNe showing constant luminosity are called plateau (SN IIP), and SNe with linear decline are called linear (SN IIL; Barbon et al. 1979). While many individual analysis have been published (e.g. Hamuy 2003), few statistical analysis of SN II have been presented, to date . Patat et al. (1994) examined some properties of 51 SN II and concluded that SN IIL have large ratio of absorption to emission $(a / e)$ of $H_{\alpha}$ P-Cygni profile values. Here, we show how the spectral and photometric properties are correlated using a large sample of high cadence and quality spectral sequences.

\section{The sample and measurements}

The sample of type II Supernovae (SNe II) employed in this study was obtained by CSP between 2004 and 2009 plus data from previous samples. From this database we selected a sub-sample of events with sufficient data to measure important spectral and photometric parameters. A large variety of SNe II are included in this sub-sample, which can be seen in the diversity of $H_{\alpha}$. Figure 1 (left) shows this variety in SNe II focusing on the $H_{\alpha}$ P-Cygni profile.

For this initial study of SNe II spectral properties we choose to focus on $H_{\alpha}$ line profiles. We measure two spectral properties: the ejecta velocity via the FWHM of emission of $H_{\alpha}$, and the ratio of EWs of absorption to emission of $H_{\alpha}$, initially proposed by Patat et al. (1994). From photometry we measure properties of the V-band light-curves (see in this edition, Anderson 2013): s1: initial decline from maximum, s2: 'plateau' decline rate (these are in V-band mags per 100 days), $M_{\text {max }}$ : maximum absolute magnitude. All the spectral measurements are interpolated to the $B_{i n f}$, defined as the time of transition between s1 and $\mathrm{s} 2$. 


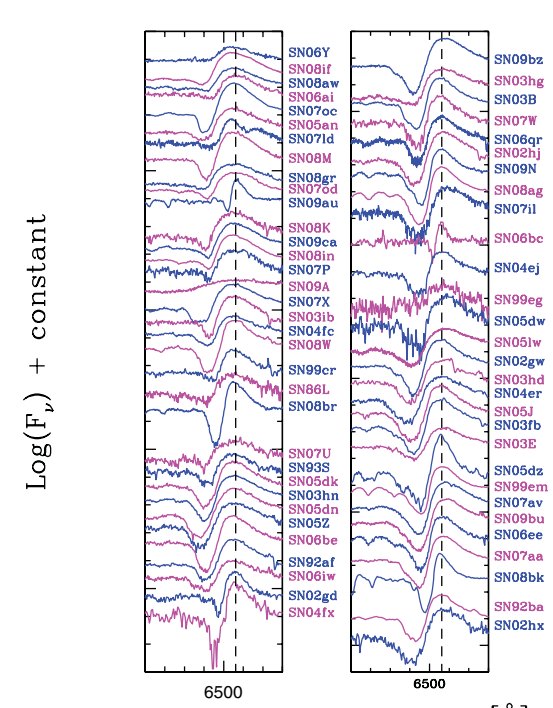

Wavelength + Constant $[\AA]$

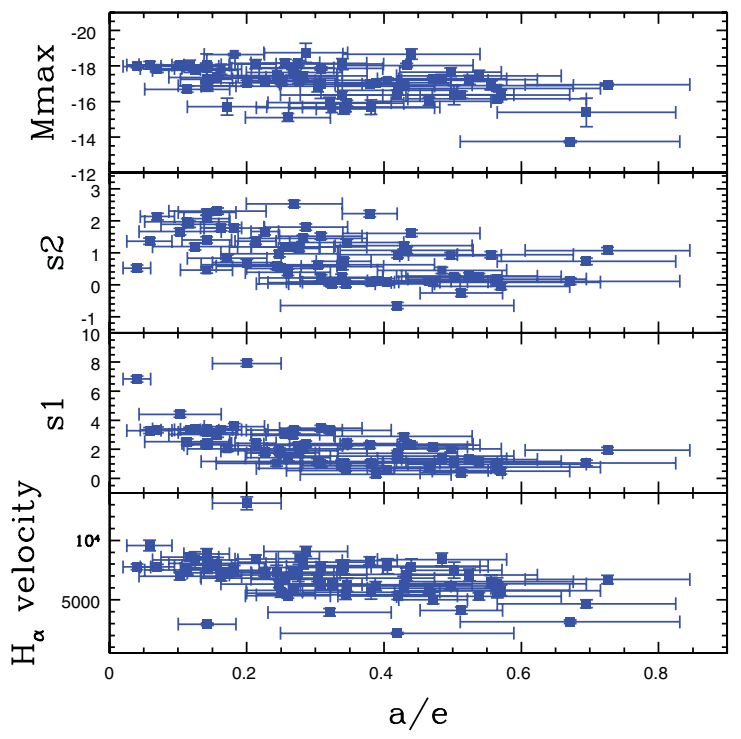

Figure 1. Left: Spectral sequences of $63 \mathrm{SNe}$ focousing on the $H_{\alpha}$ PCygni profile near $B_{\text {inf }}$. The $\mathrm{SNe}$ are ordered in terms of a/e (ratio of absorption to emission in $H_{\alpha}$ ) increasing.

Right: : Correlations between (a/e) in $H_{\alpha}$ and ejecta velocity, s1, s2 and $M_{\text {max }}$.

\section{Results}

In Figure 1 (right) we correlate $a / e$ with the ejecta velocity and three photometric parameters: s1, s2, $M_{\max }$ (corrected for $A_{v}$ using NaD lines in spectra). a/e was defined as the dominant measured parameter as it has the highest median correlation with all other parameters. This plot shows that SNe with smaller $(a / e)$ have higher $H_{\alpha}$ velocities, higher slope after the maximum and in the 'plateau' phase and are brighter. While, SNe with higher (a/e) have smaller $H_{\alpha}$ velocities, smaller slope after the maximum and in the Plateau phase, and are dimmer.

The spectral diversity found in $H_{\alpha}$ P-Cygni profiles and their correlations with light curves properties could be interpreted as circumstellar material (CSM) interaction (Pastorello et al. (2006), Inserra et al. (2011), Roy et al. (2011), Inserra et al. (2012)). However, we can not rule differences in properties such as the changes in the mass, temperature and density of the ejecta.

Acknowledgments: We want to thank to he ALMA-CONICYT 31110018 fund: "Participation of Graduate Students in international Astronomy Meetings" and the IAU Grant Allocation.

\section{References}

Anderson, J. 2013. Supernova environmental impacts, Proc. IAU Symposium No. 296 (Kolkata) Barbon, R., Ciatti, F., \& Rosino, L. 1979, A\& A, 72, 287

Hamuy, M. 2003, ApJ, 582, 905

Inserra, C., Turatto, M., Pastorello, A., et al. 2011, MNRAS, 417, 261

Inserra, C., Turatto, M., Pastorello, A., et al. 2012, MNRAS, 422, 1122

Pastorello, A., Zampieri, L., Turatto, M., et al. 2006, MNRAS, 347, 74

Patat, F., Barbon, R., Cappellaro, E., \& Turatto, M. 1994, A\& A, 282, 731

Roy, R., Kumar, B., Moskvitin, A. S., et al. 2011, MNRAS, 414, 167 